Ann. Zootech., I980, 29 (I), 3I-37.

\title{
Composition en acides aminés des protéines musculaires du porc selon le type génétique
}

\author{
P.H. IOUHIi, Régine CALMES et B. DESMOUIIN \\ Station de Recherches sur l'Elevage des Pores, \\ Centre national de Recherches zootechniques, I.N.R.A., \\ 78350 Jouy-en-Josas (France)
}

\begin{abstract}
Résumé
La composition en acides aminés des protéines du tissu musculaire a été déterminée sur 18 porcs femelles de 3 races (Landrace Français, Landrace Belge et Piétrain), à 3 stades de croissance (4o, 60 ou $80 \mathrm{~kg}$ de poids vif). vantes :

I,es teneurs moyennes, exprimées en $\mathrm{g}$ pour $\mathrm{I} 6 \mathrm{~g}$ d'azote, à $80 \mathrm{~kg}$ de poids vif, sont les sui-

\begin{tabular}{|c|c|c|c|c|}
\hline Hypro & $: \quad \mathbf{I}, \mathbf{O}$ & Asp : 10,1 ; & Thr : 4,7 ; & Ser : 4,05; \\
\hline Glu & : I 5,25; & Pro: 4,6 ; & Gly : 5,o ; & Ala : 5,9 \\
\hline Val & $: \quad 5,45$ & Ileu : & Leu : 8,25; & Tyr : 3,6 ; \\
\hline Phe & $: \quad 4,05$ & His : 3,95 & Lys : 8,4 ; & Arg : 6,4 ; \\
\hline Cys & : 1,2 ; & Met : 2,85 & & \\
\hline
\end{tabular}
\end{abstract}

L'amplitude des variations est faible, selon le stade de croissance; seule une augmentation de certains acides aminés indispensables ( $\mathrm{L}, \mathrm{ys}$, Thr, Met et Phe) est constatée, principalement entre 40 et $60 \mathrm{~kg}$ de poids vif. Par ailleurs, l'expérience ne permet pas de mettre en évidence des différences de composition entre type génétique.

En termes de dépôts journaliers, des différences observées entre les types génétiques et leurs répercussions sur le besoin correspondant sont également discutées.

\section{Introduction}

L'apport nutritionnel doit être modulé selon les potentialités de production (croissance en particulier) de l'animal considéré. Ceci est particulièrement vrai quand on considère l'apport qualitatif en protéines dans l'alimentation du porc (DUEE et SEVE, I978), mais peu d'éléments permettent actuellement de proposer des recommandations selon le type génétique recouvrant différentes potentialités de croissance ou de dépôts musculaires. 
Ainsi, a-t-on souligné, à la suite d'une expérience portant sur la relation entre l'apport nutritionnel et le type génétique (SEILIER et al., r974; Desmoulin et POMMERET, I974) une réponse à l'élévation des apports protéiques dans la ration, plus prononcée chez le porc femelle de type hypermusclé (Landrace Belge) que chez le pore de type normal (Landrace Français). Une investigation plus poussée est, à ce stade, nécessaire pour définir les besoins nutritionnels propres à chaque type génétique. L'analyse chimique corporelle peut alors représenter une voie d'approche complémentaire, tout en sachant que les résultats acquis par cetta méthode ne sont que le reflet apparent des processus d'anabolisme et de catabolisme protéiques.

C'est dans ce but que l'on a procédé à l'analyse des teneurs en acides aminés du muscle, à différents stades de la croissance de porcs de trois types génétiques (Landrace Français, Landrace Belge et Piétrain), dans le cadre de l'expérience précédemment citée.

\section{Matériel et méthodes}

L'expérience porte sur I 8 pores femelles des trois races considérées, abattues à l'un des trois stades d'abattage suivants : 40, 60 et $80 \mathrm{~kg}$ de poids vif, soit deux animaux par point expérimental.

A partir d'un poids moyen de $26 \mathrm{~kg}$, tous les animaux reçoivent, d'une façon libérale, le même régime à base d'orge et de tourteau de soja, apportant 2985 Kcal d'énergie digestible par $\mathrm{kg}, 20,0 \mathrm{p}$. Ioo de matières azotées et I, I $5 \mathrm{p}$. Ioo de lysine. A l'abattage, une découpe des carcasses, suivie d'une dissection anatomique individualisant 6 compartiments tissulaires dans chaque pièce permet d'isoler la quantité totale de muscles représentant la "viande maigre " de référence. Les analyses chimiques sont alors effectuées sur le produit lyophilisé d'un homogénat des muscles totaux (DESMOULIN et POMMERE'T, I974).

I,es teneurs en acides aminés des muscles sont déterminées sur cet échantillon lyophilisé, après hydrolyse acide (24 et $48 \mathrm{~h}$ avec $\mathrm{Hcl}(6 \mathrm{~N})$ ), suivant la méthode de MoORE et al., (I958), utilisant la technique de chromatographie sur colonne échangeuse d'ions. Le tryptophanc, totalement détruit lors de l'hydrolyse acide, n'est pas dosé; les acides aminés soufrés sont oxydés au préalable par l'acide performique et dosés par la même méthode que précédemment.

\section{Résultats et discussion}

Dans le tableau I, sont consignés 1es performances de croissance et les riveaux de consommation des animaux étudiés. Ėn se référant à l'étude de SEILIER et al., (I974), on remarquera que l'échantillon est bien représentatif de chaque race pour les porcs de type Landrace (Français ou Belge). Ceci est moins le cas pour les porcs Piétrain, dont la vitesse de croissance est plus hétérogène entre stades et notamment plus élevée à $80 \mathrm{~kg}$ que celle relevée dans un échantillon plus important (SELIIER et al., I 974).

En ce qui concerne les teneurs en acides aminés dans le muscle (tabl. 2), on constatera d'une manière générale que l'amplitude des variations est faible, 
TABI_EAU I

Croissance et consommation des animaux(1) Growth rate and feed intake of animals

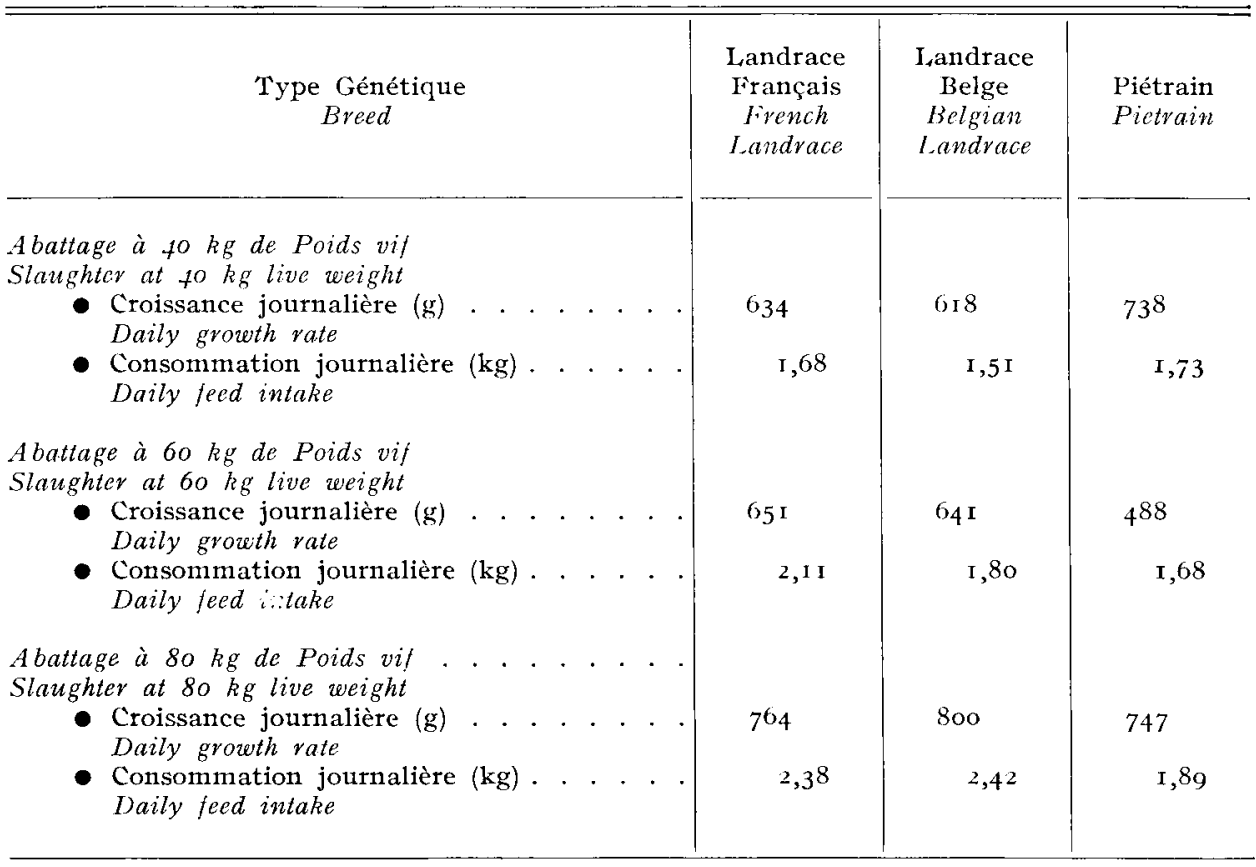

(1) A partit de $26 \mathrm{~kg}$ de poids vif (From $26 \mathrm{~kg}$ live weight).

illustrée par un cœfficient de variation de I à $8 \mathrm{p}$. Ioo aux stades considérés. La teneur en hydroxyproline, en très faible quantité dans les échantillons, est, par contre, beaucoup plus variable.

Si l'on compare l'évolution des concentrations en acides aminés indispensables en fonction du poids vif, pour chaque type génétique, on notera une augmentation sensible de certains d'entre eux (Lysine, Thréonine, Méthionine et Phenylalanine) entre 40 et $60 \mathrm{~kg}$ de poids vif pour les porcs de type Landrace Français et Piétrain. Pour le Landrace Belge, cette augmentation semblerait plus tardive (entre 60 et $80 \mathrm{~kg}$ ). Cet enrichissement pourrait signifier un accroissement relatif dans le muscle des protéines myofibrillaires.

Pour les acides aminés non indispensables, il ne semble pas non plus y avoir de règle générale quant à leur évolution en fonction du poids vif. A cet égard, il apparaît plutôt une constance des teneurs en proline, glycine et hydroxyproline, acides aminés caractéristiques des protéines du collagène.

A $80 \mathrm{~kg}$ de poids vif, les teneurs moyennes en acides aminés du muscle sont tout à fait comparables à celles rapportées par OSLAGE et SchULz (I977), mais sont légèrement inférieures à celles analysées par PION et FAuConnEAU (I966), principalement pour la lysine et l'histidine. Dans ce dernier cas, il est vraisemblable que la présence dans le muscle de constituants azotés non protéiniques ( $\beta$ alanylhistidine, principalement) en surestime la valeur. 


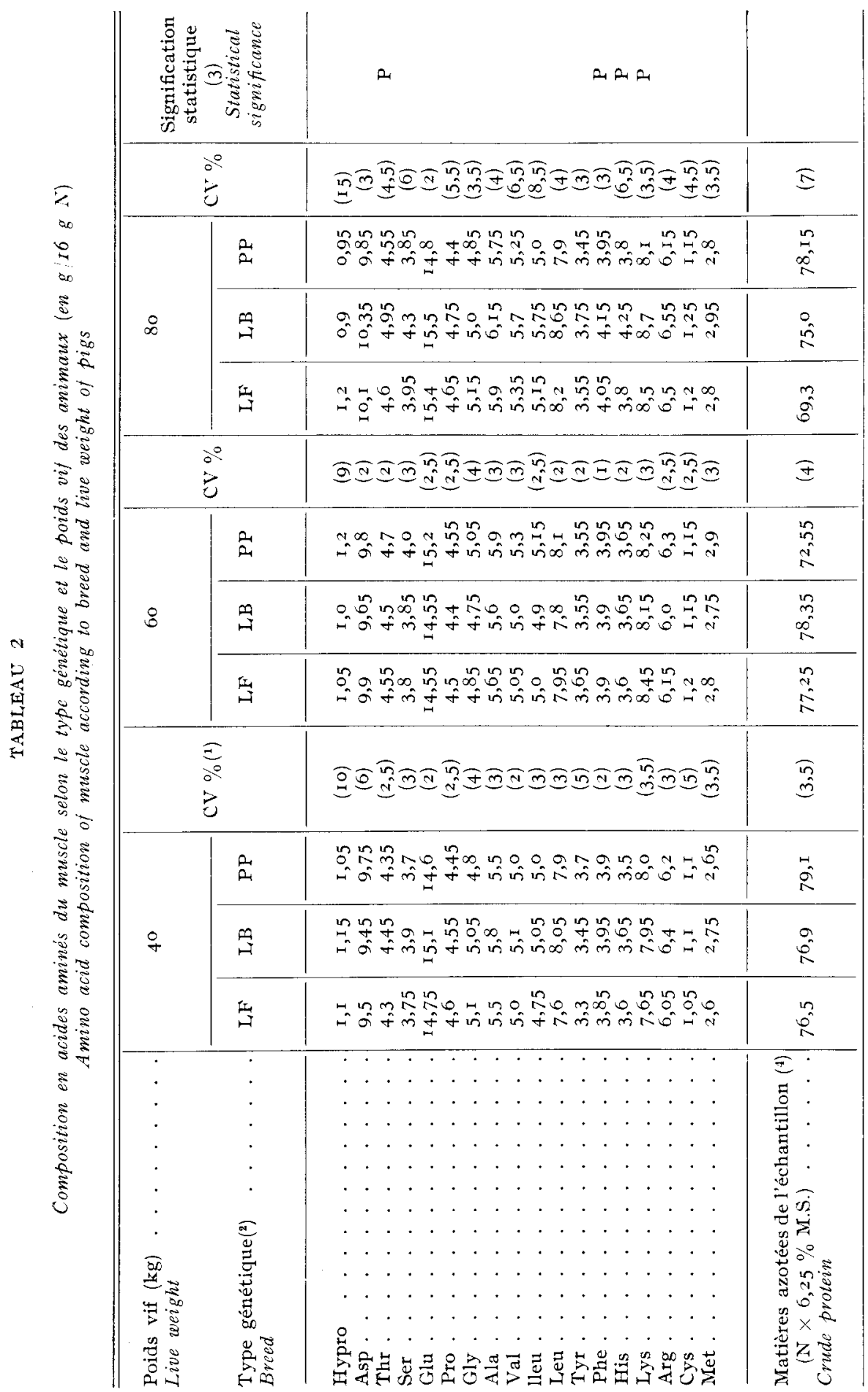


Par ailleurs, la composition en acides aminés du muscle apparaît constante d'une race à l'autre, si l'on excepte, chez le Piétrain, une tendance à la diminution des teneurs en certains aciđes aminés non indispensables (Hypro, Gly, Pro), au stade ultime d'observation, confirmant ainsi les résultats antérieurs de SEr.LIER et BocCARD (I97I).

Par rapport at1 veau (PIVA et Guglifi,Metri, I979), le tissu musculaire du porc semble plus riche en certains acides aminés indispensables (Histidine, Thréonine, Valine, Isoleucine, Leucine), mais plus pauvre en acide glutamique, quoique, selon LAWRIE (I979), les différences de composition entre espèces soient négligeables.

Les quantités de protéines ou d'acides aminés (la lysine, par exemple) déposées dans le compartiment musculaire sont calculées compte tenu des quantités de muscles mesurées par dissection et dont 1'évolution moyenne a déjà été rapportée (Desmoulin et Pommeret, I975). Les types génétiques se différencient alors fortement : par rapport au Landrace Français, les pores de type hypermusclé présentent, à $80 \mathrm{~kg}$ de poids vif, un accroissement des quantités de protéines musculaires de 7 p. Ioo (Landrace Belge) jusqu'à près de 30 p. Ioo (Piétrain). L'augmentation des quantités de lysine dans le muscle est également comparable (tabl. 3). Le dépôt quotidien en protéines et en lysine dans le tissu musculaire, en tena nt

\section{TABIEAU 3}

Dépot musculaive en protéines et en lysine entre 40 et $80 \mathrm{~kg}$ de poids vif Crude Protein and Lysine deposition in muscles between 40 and $80 \mathrm{~kg}$ live weight

\begin{tabular}{|c|c|c|c|}
\hline $\begin{array}{l}\text { Type Génétique } \\
\text { Breed }\end{array}$ & $\begin{array}{l}\text { Landrace } \\
\text { Français } \\
\text { French } \\
\text { L.andrace }\end{array}$ & $\begin{array}{l}\text { Landrace } \\
\text { Belge } \\
\text { Belgian } \\
\text { Landrace }\end{array}$ & $\begin{array}{l}\text { Pietrain } \\
\text { Pietrain }\end{array}$ \\
\hline $\begin{array}{l}\text { Gain moyen quotidien entre } 40 \text { et } 80 \mathrm{~kg}(\mathrm{~g}) \\
\text { Daily growth vate } 40-80 \mathrm{~kg}\end{array}$ & 833 & 833 & 749 \\
\hline $\begin{array}{l}\text { Protéines totales des muscles }(\mathrm{kg}) . . . \\
\text { Total crude protein in muscles }(\mathrm{kg}) \\
\text { Lysine totale des muscles }(\mathrm{kg}) . . . \\
\text { Total lysine in muscles }(\mathrm{kg})\end{array}$ & 5,92 & $\begin{array}{r}6,35 \\
0,55\end{array}$ & $\begin{array}{l}7,6 \mathrm{I} \\
0,62\end{array}$ \\
\hline $\begin{array}{l}\text { Dépot protéique moyen }(\mathrm{g} / \mathrm{j}) \\
\text { Mean crude Protein deposition }(\mathrm{g} / \text { day }) \\
\text { Dépôt en lysine moyen }(\mathrm{g} / \mathrm{j}) \cdot \cdot \cdot \cdot \cdot \cdot \\
\text { Mean lysine deposition }(g / \text { day })\end{array}$ & $\begin{array}{l}60 \\
5,6\end{array}$ & 66 & $\begin{array}{r}80,5 \\
6,5\end{array}$ \\
\hline $\begin{array}{l}\text { Lysine ingérée }(\mathrm{g} / \mathrm{j}) \\
\text { Lysine intake }(\mathrm{g} / \mathrm{day}) \\
\text { Iysine déposée }(\%) \\
\text { Iysine ingérée }\end{array}$ & $\begin{array}{l}3 \mathrm{I}, \mathrm{I} \\
\mathrm{I} 8,2\end{array}$ & $\begin{array}{l}3 \mathbf{I}, 2 \\
20, I\end{array}$ & $\begin{array}{l}23,2 \\
28,7\end{array}$ \\
\hline
\end{tabular}


compte des valeurs obtenues à $40 \mathrm{~kg}$ de poids vif et de la vitesse de croissance ultérieure, souligne parallèlement des différences d'utilisation selon les races. Pour un même niveau de croissance et dans des conditions libérales de consommation d'aliment, les porcs Landrace Belge déposent ro p. Ioo de lysine en plus, entre 40 et $80 \mathrm{~kg}$ de poids vif. Dans le cas du Piétrain l'accroissement est plus prononcé, mais il peut être surestimé, si l'on considère le niveau de croissance des animaux étudiés et le choix de la période d'étude (entre 40 et $80 \mathrm{~kg}$ ) favorisant les porcs de cette race (DESMOULIN et POMMERET, I975). Quoi qu'il en soit, cette potentialité différente de croissance musculaire entre les deux types de Landrace pourrait correspondre à des besoins protéiques différents. Səlon WIESEMürLER et POPPE (I974), le pourcentage de lysine ingérée qui est déposée dans la carcasse est voisin de $40 \mathrm{p}$. I 00 dans des conditions optimales d'alimentation mais peut d'ailleurs être plus élevé (WIESEMüLLER et al., I978). Ce rendenent en lysine est bien évidemment plus bas, dans la présente étude, compte tenu des quantités pléthoriques de lysine consommée. En supposant une utilisation de la lysine optimale et comparable pour les deux types de Landrace, il ressortirait donc un accroissement du besoin chez le Landrace Belge, au minimum de $\mathrm{I}, 5 \mathrm{~g}$ par iour, soit environ ro p. Ioo de plus que les normes préconisées à ce stade.

Cette analyse permettrait ainsi de proposer une base théorique d'évaluation du besoin en acides aminés en fonction du type génétique. Elle n'exalut pas, bien au contraire, des mesures directes impliquant l'étude d'autres paramètres.

Accepté pour publication en février 1980.

\section{Summary}

\section{Amino acid composition of muscle crude protein according to the pig breed}

The amino acid composition of muscle crude protein was determined in 18 female pigs of three breeds (French Landrace, Belgian Landrace and Pietrain), distributed into three groups according to the slaughter weight $(40,60$ or $80 \mathrm{~kg}$ live weight).

The mean levels of amino acids (expressed in $\mathrm{g}$ per $\mathrm{I} 6 \mathrm{~g}$ total nitrogen at $80 \mathrm{~kg}$ live weight) were the following :

\begin{tabular}{|c|c|c|c|c|c|}
\hline Hypro & J.0 ; & Asp & IO.1 ; & Thr : 4.7 ; & Ser $: 4.05$ \\
\hline Glu & : I5.25; & Pro & 4.6 & Gly : 5.0 ; & Ala : 5.9 \\
\hline Val & $: \quad 5.45$ & Ileu & $5 \cdot 3$ & Leu : 8.25; & Tyr: 3.6 \\
\hline Phe & 4,05 & His & $3.95 ;$ & Lys : 8.4 ; & Arg : 6.4 \\
\hline Cys & I.2.; & Met & 2.85 & & \\
\hline
\end{tabular}

The magnitude of the variations according to slaughter weight was low. Only an increase in some essential amino acids (Lys, Thr, Phe and Met) was observed, mainly between 40 and $60 \mathrm{~kg}$ live weight. No difference according to genotype was found in the amino acid composition of the muscle.

In terms of daily amino acid deposition, the differences mainly depended on differences between breeds in the deposition of protein. Thus, at similar growth rates and in liberal feeding conditions, the lysine deposition of Belgian Landrace pigs as compared to French Landrace ones is ro per cent higher between $4^{\circ}$ and $80 \mathrm{~kg}$ live weight. 


\section{Références bibliographiques}

Desmotin B., Pommeretr P., i974. Références de composition anatomique et critères de classification des carcasses de porcs femelles des types Landrace Français, Landrace Belge et Piétrain. Journées Rech. Porcine en France, 6, 22I-232, I.N.R.A.-I.T.P., éd., Paris.

Insmoulin B., Pommerit P., I975. Fvolution de la composition anatomique des porcs femelles des types Landrace Français, Landrace Belge et Piétrain : conséquences sur les méthodes d'orientation des productions. Journées Rech. Porcine en Irance, 7, I 79-I94, I.N.R.A.-I.T.P., éd., Paris.

Drki: P. H., SEVE B., I978. Alimentation azotée du porc. Journées Rech. Porcine en France, 10, $167-207$, I.N.R.A.-I.T.P., éd., Paris.

LAwkif R, A., I 979. In Meat Science, 368, Pergamon Int. Library.

MOCRE S., SPACKMAN D. H., STEIN W. H., I958. Chromatography of amino acids on sulfonated polystyrene resins. Anal. Chem., 30, I I 85-I 190 .

OsiagF, H. J., Schulz E., 1977. Aminosaürenansatz in verschiedenen Organen und im Gesamtkörper wachsender schweine sowie Ableitungen zum Aminosaürenbedarf. I'th International Symposium on Amino Acids, Budapest.

Pion R., Fauconneat G., i966. Les acides aminés des protéines alimentaires. Méthodes de dosage et résultats obtenus. Cahier A.L.C., no 6, I57-175, Commentry.

PIVA ( $\dot{ }$., GUGLIELMFTTI D., 1979. Meat amino acid composition of calves and young bulls slaughtered between $200 \mathrm{~kg}$ and $500 \mathrm{~kg}$ live weight. Ann. Fac. Agrar., 19, JoI-Io8.

Sellifr P., Boccaris R, I97I. Ftude du taux d'hydroxyproline d'un muscle et de ses relations avec certaines caractéristiques de composition corporelle dans les races Large White et de Piétrain. Ann. Génét. Sél. anim., 3, 433-448.

Seltier P., Hotix Y., Desmoulin B., HeNRy Y., i974. Premières observations sur la relation entre conditions nutritionnelles et type génétique chez des porcs femelles. Journées Rech. Porcine en France, 6, 209-2I9, I.N.R.A.-I.T.P., éd., Paris.

WIEslimüliter W., Pol'es., I 974. Untersuchungen zum Aminosaürenbedarf von Mastschweinen (Börge) 5. Einfluss der Aminosaürenernährung auf den Proteinansatz. Arch. Tierernähr., 24, 535-542.

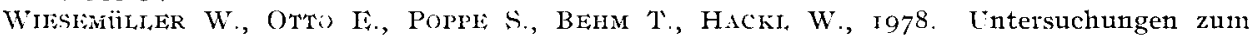
leistungsabhangigen 1 rsinbedarf ron Mastschweinen 3 . Einfluss des Aminosaüren und Energieverzehrs anf den Fett, Eiweiss- und Lysinansatz der Schweine. Arch. Tierernähr., 28, $305-316$. 